\title{
CONGENITAL ACUTE MEGAKARYOCYTIC LEUKEMIA (AML-7)
}

\author{
Ananita Novia Silviarianti ${ }^{1}$, Arifoel Hajat $^{2 *}$ \\ ananita.dr@gmail.com
}

${ }^{1}$ Clinical Pathology Specialization Programme, Department of Clinical Pathology, Medical Faculty Airlangga University, Dr. Soetomo Hospital, Mayjen. Prof. Dr. Moestopo st no. 6-8 Surabaya, 60286, Indonesia

${ }^{2}$ Department of Clinical Pathology, Medical Faculty Airlangga University, Dr. Soetomo Hospital, Mayjen. Prof. Dr. Moestopo st no. 6-8 Surabaya, 60286, Indonesia.

\section{Abstract}

\begin{abstract}
Background: Congenital Leukemia (CL) is a disorder that is very rare in newborns, most often originate from the myeloid series M4 / M5. Peripheral blood showed mononuclear cells with cytoplasmic bleb which is sometimes very difficult to distinguish between myeloid and lymphoid series. Acute megakaryocytic leukemia is a rare type of AML with an incidence of 0.5 per million per year and may accompanied by Down Syndrome. We report an AML-M7 case in a nenonate with Down syndrome.
\end{abstract}

Case Description: A 3-day-old neonate was referred from RSIA Amanah Probolinggo with a suspected diagnosis of congenital leukemia and Down syndrome and main complaint of vomiting. Physical examination: Blood pressure $72 / 31 \mathrm{mmHg}$, pulse $137 \mathrm{x} / \mathrm{min}$; breathing $40 \mathrm{x} /$ minute; temperature $36.5^{\circ} \mathrm{C}$, body weight $2,700 \mathrm{~g}$, height $46 \mathrm{~cm}$, head circumference $33 \mathrm{~cm}$, jaundiced sclera, chest retraction, papular macular rash at abdomen, simian crease. Clinical chemistry: sodium $118 \mathrm{mmol} / \mathrm{L}$, total bilirubin 7.36, CRP 11.6. Duodenal BOF atresia and duodenal web. Hematology: Hb 17.6 g/dl, MCV 90.4 fL, MCH 32.4 pg, MCHC $35.8 \mathrm{~g} / \mathrm{dl}$, WBC $58.00 \times 10^{3} \mu \mathrm{L}$, platelets $74 \times 10^{3} / \mu \mathrm{L}$, IPF $25.8 \%$. Peripheral blood smear: Leukocytosis with $70 \%$ megakaryoblasts. Specific immunophenotyping against megakaryocytes CD $36(+)$ and myeloid CD 33, CD 34, CD 117 series with CD7 aberration. The patient died at the age of 8 days.

Discussion: Neonates with a clinical manifestasion of Down Syndrome, morphology of peripheral blood smear dominated by megakaryoblasts, increased IPF and immunophenotyping CD $36(+)$ help diagnose Congenital Acute Megakaryocytic Leukemia.

Conclusion: AML-M7 is a congenital leukemia which is established based on blood smears and immunophenotyping and is related to Down Syndrome.

Keywords: Congenital Leukemia, AML-M7, Down Syndrome: 


\section{Introduction}

Neonatal Leukemia is defined as occurring within the first 28 days of life and is mostly congenital and rare. In two-thirds of patients the disease manifests as AML with monocytic/monoblast characteristics and the remainder develops ALL. (1) Acute megakaryocytic leukemia (AML-M7) is a type of AML that is $50 \%$ found in children with Down syndrome. (2) Recent reports have shown that children with Down syndrome syndrome and AML, especially AMKL have a very high cure rate with survival rates ranging from $80-100 \%$. (3) Morphologically of AML-M7 megakaryocytes are difficult to distinguish from lymphoblasts or normoblasts because they both have a cytoplasmic blep, so we need immunophenotyping. (4) AML M7 showed positive CD61, CD41, CD36, CD33, positive-negative on CD34, MPO and negative on lymphoid lineage.

Based on a case report reported by Ananita Novia Silviarianti, a neonate with the peripheral blood cell picture showed the presence of mononuclear cells with cytoplasmic bleps leading to AML-M7, an immunophenotyping profile is needed to establish the diagnosis of AML-M7.

\section{Case Report}

3 days old neonate with chief complaint vomit. The patient has been vomiting since the first day and also hardening of the stomach.

The patient is a referral from a hospital in Probolinggo. History of birth by Caesarean section, birth weight 2700 grams, length $46 \mathrm{~cm}$, AS 5-6. There was a history of premature rupture of membranes 6 hours before. Physical examinations: Weak, compos mentis (Incubator), Blood pressure 72/31 mmHg, pulse 137 $\mathrm{x} / \mathrm{min}$; breathing $40 \mathrm{x} /$ minute; temperature $36.5^{\circ} \mathrm{C}, \mathrm{SpO} 2: 99 \%$, body weight $2,700 \mathrm{~g}$, height $46 \mathrm{~cm}$, head circumference $33 \mathrm{~cm}$, anemic conjunctiva, jaundiced sclera, chest retraction, papular macular rash at abdomen, simian crease. Clinical chemistry: sodium $118 \mathrm{mmol} / \mathrm{L}$, total bilirubin 7.36, CRP 11.6. Duodenal BOF atresia and duodenal web. Hematology: Hb 17.6 g / dl, MCV 90.4 fL, MCH 32.4 pg, MCHC $35.8 \mathrm{~g} / \mathrm{dl}$, WBC 58.00 x $103 \mu \mathrm{L}$, platelets 74 x $103 / \mu \mathrm{L}$, IPF $25.8 \%$.

The results of the peripheral blood cell showed Normochromic normochromic erythrocyte morphology anisopoikilocytosis. Leukocytosis with abnormal megakaryoblasts $60 \%$. Suspected AML-M7 dd ALL Suggestion: BMA, Immunophenotyping (figure 1).

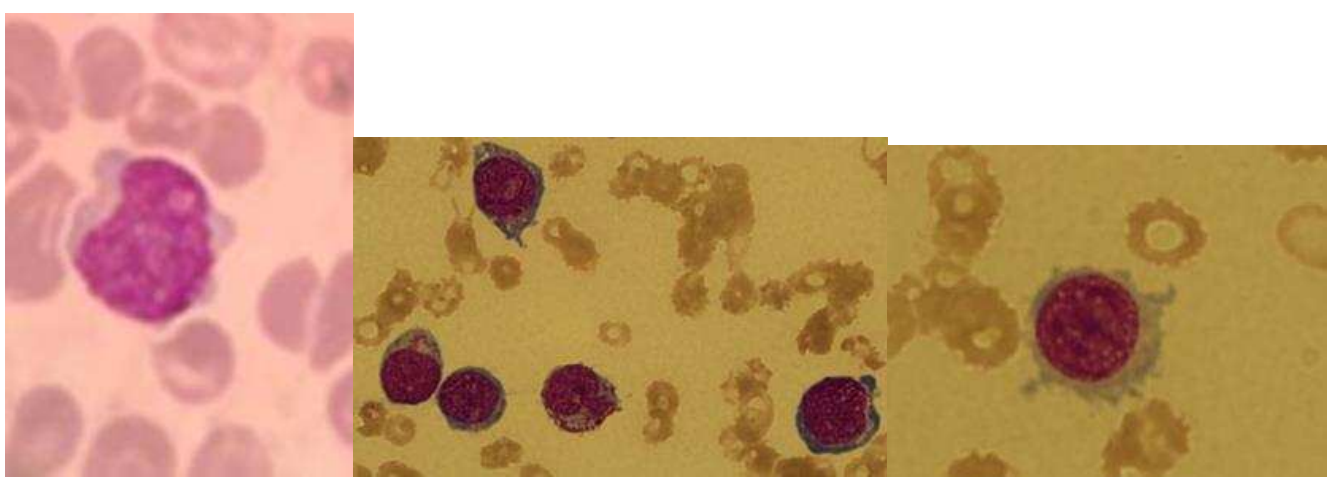

Figure 1. Abnormal morphology of micromegakaryocytes in peripheral blood picture 
Immunophenotyping was positive for $\mathrm{CD} 33, \mathrm{CD} 34 \mathrm{CD} 36, \mathrm{CD} 117$ and $\mathrm{CD} 7$ (table 2). Immunophenotyping conclusion Myeloid lineage according to AML-M7 with aberrations CD7, CD 36 (+), GLY A (-). Clinical chemistry examination sodium 118, potassium 2.8, chloride 76 , an increase in BUN 30, creatinine 2.6, total bilirubin 7.36, CRP 11.6, phosphate 7.1, uric acid 13.9. The APTT and PTT values were elongated 63.2 and 18.2.

Table 1. Immunophenotyping result

\begin{tabular}{|c|l|}
\hline Marker & Hasil \\
\hline CD 33 & Positive \\
\hline CD 34 & Positive \\
\hline CD 36 & Positive \\
\hline CD 117 & Positive \\
\hline CD 7 & Positive \\
\hline CD 13 & Negative \\
\hline CD 61 & Negative \\
\hline GLY A & Negative \\
\hline
\end{tabular}

The patient was diagnosed with Suspected Neonatal Megacryocytic Leukemia and Suspected Down

Syndrome by the clinician and received supportive therapy. The patient's treatment was stopped because the patient died at the age of 8 days.

\section{Discusion}

Congenital Leukemia is the term used for leukemia that is diagnosed at birth or within the first month of life and is a rare case.(5) A study in the north of England reported an incidence of between 4.3 and 8.6 per million live births.(6) AML-M7 or AMKL is a very rare type of AML that represents about $1 \%$ of all childhood leukemias, with an incidence of 0.5 per million per year.(2) This case from day one have the diagnostic criteria for congenital leukemia which is include a ) presentation of disease at or immediately after birth (<30 days), b) immature white blood cell proliferation, c) infiltration of cells into extrahematopoeitic tissue, and d) absence of conditions mimicking Congenital Leukemia as in congenital syphilis, and not found blood group incompatibility, TORCH infection.(7) Most Congenital Leukemias originate from the myeloid lineage, whereas leukemia in children is usually b of lymphoid origin.(7)

About half of congenital AML is M4 or M5, there is no sex predominance but there is ethnic variation with a higher incidence in Asians and Hispanics. The median age of presentation is 6 years, ranging from 3 months to 16 years, but $40 \%$ of cases have been seen in children 41 months of age or younger. (8) No chromosomal abnormality examination was carried out in this case, only the clinician 
diagnosed as a Down Syndrome based on the patient's clinical appearance, the presence of a distinctive face in Down Syndrome and the finding of Simian Crease. Acute Myeloblastic Leukemia (AML) M7 is a subtype of AML according to the FAB. The diagnosis of AML-M7 was defined as the number of megakaryoblasts $>50 \%$ of the total number of blasts $(>20 \%)$ found in blood smear evaluation or bone marrow aspirate. (9) Blood rutine results of this patient showed $\mathrm{Hb} 17.6 \mathrm{~g} / \mathrm{dL}$, WBC 58,000/ $\mu \mathrm{L}$, PLT 74,000/ L, IPF 25.8\%. Low platelets and high leukocytes indicate leukemia.

Clinical signs of leukemia may be obvious at birth, including hepatosplenomegaly and ecchymosis. Infiltration of leukemic cells into the skin (leukemia cutis / chloroma) is commonly found when the disease appears early in life and is also seen in stillbirths. with leukemia. (10) At birth many infants have respiratory distress either due to leukemic infiltration of the lungs or atelectasis. In infants who suffer from the disease in the first month (not at birth), the symptoms are not clear with mild fever, diarrhea, hepatomegaly but Leukemia cutis is very rare. (10) This case was a premature baby who was born with complaints of weakness with AS 5-6, abdominal distended and then vomiting greenish in color, also had ecchymosis in the abdominal region and difficulty breathing. On physical examination, icteric sclera and Simian Crease were found in the extremity region.

In this case also obtained a high value on the Immature Platelet Fraction (IPF). Thrombocytopenia in leukemia patients is caused by bone marrow suppression, which causes decreased platelet production and low IPF values. However, in this case, a high IPF value was obtained, so the suspicion of immature platelets could not be ruled out. The morphological features of megakaryocytes described by Naeim et al. Megakaryocytes are very pleomorphic with small to large cell shapes, round with much cytoplasm, clear nucleus and hard to find and often have blep cytoplasm. The blood smear evaluation results in this case showed a small mononuclear cell with bleeped cytoplasm, loose chromatin and an indistinct nucleus which suspected an abnormal micromegakaryocyte or lymphoblast with the conclusion suspicious an AML-M7 dd ALL.

BMA was not performed on this patient due to the patient's condition that did not allow it, so to help confirm the diagnosis, an immunophenotyping examination was carried out. Several markers were tested to narrow the diagnosis. Myeloid lineage was seen at CD33, CD13, CD117, and MPO. Lymphoid lineage was seen on CD7 and CD3. The markers for megakaryocytes were assessed at specific CD36 and CD61. The results of the immunophenotyping examination in this case were positive on CD 33, CD 36, $\mathrm{CD} 34, \mathrm{CD} 117$ and $\mathrm{CD}$ 7. The results were negative on CD13, CD61, GLY A and MPO. Immunophenotyping that describes megakaryocytes is the expression of CD 41, CD 61, factor VIII, CD 36, CD 38, CD 7 aberrations, negative on myeloid markers including MPO, CD 34 and CD 1174. Immunophenotyping that is specific for platelet antigens is the expression of CD41, CD61, CD364.

The CD61 marker, also known as GPIIIa, is a glycoprotein found on platelets, monocytes, endothelial cells, smooth muscle, and mast cells. This protein is encoded by the Integrin Beta-3 (ITGB3) gene. CD61 has a role in platelet aggregation as well as a receptor for fibrinogen and von Willbrand factor. Until now, CD61 has been used as an immunophenotyping marker to assess megakaryocyte leukemia. (9) CD36 marker also known as platelet glycoprotein 4 (GPIV) is a protein that is encoded by the CD36 gene. CD36 is an integral membrane protein found on the surface of platelets. Together with CD41 and CD61, CD36 is used as an immunophenotyping marker in the diagnosis of AML-M79.

Negative result for the lymphoid marker CD3, CD79a could rule out the presence of lymphoblasts. A negative result on CD 13 excludes the diagnosis as M4-M5. The suspicion of AML was strengthened by positive immunophenotyping on the myeloid lineage markers, namely CD33, CD117. Negative results were obtained on CD 61 but positive results were obtained on another megakaryocyte marker, namely CD36, thus further strengthening the diagnosis of AML-M7. 
According to Campana et al, the probability of the appearance of myeloid markers in AML-M7 namely CD33, CD13 and CD117 is around 75\%, 30\% and 30\%. (11) Martin et al also reported positive myeloid lineage values in AML-M7. (12)

\section{Conclusion}

Down Syndrome and the onset of disease, routine blood results, peripheral blood smears and immunophenotyping leads to Congenital Acute Megakaryocytic Leukemia (AML-M7).

\section{Acknowledgements}

The author would like to thank Mr. Arifoel Hajat, MD., who has helped and guided the writing of this case report.

\section{References}

1. Irene Roberts., Nicholas J. Fordham., Anupama Rao., Barbara J. Bain., Neonatal leukaemia, British Journal of Haematology, 2018, 182, 170-184

2. N. B. Mathur., Neha Joshi., T. Singh., Meeta Singh., Congenital acute megakaryocytic leukemia, Indian Journal of Medical and Paediatric Oncology 2011;32:165-7

3. Dharini Gandhi., Jigna Patel., Case Reports: Acute megakaryocytic leukemia (m7) in a newborn with down syndrome, International Archives of Integrated Medicine, Vol. 6, Issue 6, June, 2019

4. Faramaz N., P. Rao, Sophie S., Ryan P., Atlas of Hematopathology 2nd Edition. Elsevier. 2018. 273-276

5. Aishwarya Raj., Sewali Talukdar., Smita Das., Pabitra Kumar Gogoi., Congenital Leukemia, Indian J Hematol Blood Transfus (Sept 2014) 30

6. Bajwa RP, Skinner R, Windebank KP, Reid MM. Demographic study of leukaemia presenting within the first three months of life in the northern health region of england. J clin Pathol 2004;57: 186-8

7. Mccoy JP Jr, Overton wR. Immunophenotyping of congenital leukemia. cytometry 1995; 22:85-8

8. Prakash Kp., Rau Atk., Bhat St., Rau Ar., Congenital Leukemia A diagnostic dilemma, Indian J Med Paediatr Oncol 2008 4:41-43

9. Kaushansky K., Beutler E., Lichtman M. A., Kipps T. J., Prchal J. T., Seligsohn U., Williams Hematology 8th Edition Volume 1. New York, Mc Graw Hill. 2010; 193-229

10. Campwell WA., Storlazzi E, Vintizileos AM., Fetal neonatal leukemia. Arch Dis Child 37:93-98

11. Campana D., Behm F.G., Immunophenotyping of Leukemia. Journal of Immunological Method. 2000;5975

12.Tallman M.S., Neuberg D., Bennett J.M., Francois C.J., Paietta E., et al. Acute Megakaryocytic Leukemia: The Eastern Coorperative Oncology Group Experience. Blood 2000; 96:2405-2411 